\title{
MODELING THE EFFECT OF A NOVEL AUTO-DISSEMINATION TRAP ON THE SPREAD OF DENGUE IN HIGH-RISE CONDOMINIA, MALAYSIA
}

\author{
DAVID GREENHALGH* and YANFENG LIANG \\ Department of Mathematics and Statistics \\ University of Strathclyde, Glasgow G1 1XH, UK \\ *david.greenhalgh@strath.ac.uk \\ WASI AHMAD NAZNI and GUAT-NEY TEOH \\ Medical Entomology Unit, Institute for Medical Research \\ Jalan Pahang, 50588 Kuala Lumpur, Malaysia \\ HAN LIM LEE \\ Vector-Borne Disease Control Branch \\ Disease Control Division, Ministry of Health \\ Putrajaya, Malaysia \\ EDUARDO MASSAD \\ School of Applied Mathematics, Fundacao Getulio Vargas \\ Rio de Janeiro, Brazil \\ Department of Legal Informatics, School of Medicine \\ University of Sao Paulo and LIM 01/HCFMUSP \\ Av. Dr. Arnaldo 455, Sao Paulo, SP01246-903, Brazil \\ London School of Hygiene and Tropical Medicine \\ London, UK
}

Received 2 August 2018

Accepted 8 November 2018

Published 20 December 2018

\begin{abstract}
In this paper, we use the classical Ross-Macdonald model to analyze the effect of the Mosquito Home System (MHS), which is an example of an auto-dissemination trap, in controlling the spread of dengue in Malaysia in a high-rise condominium environment. By using the national dengue data from Malaysia, we are able to estimate $\lambda$ which represents the initial growth rate of the dengue epidemic and thus allows us to estimate the number of mosquitoes in Malaysia. The basic reproduction number $R_{0}$ is also obtained. We have constructed a mathematical expression which allows us to estimate the potential number of breeding sites for Aedes mosquitoes. Later on, by using the data available from the 11 months trials carried out in three blocks of flats in Selangor, we improved on our dengue
\end{abstract}

* Corresponding author.

This is an Open Access article published by World Scientific Publishing Company. It is distributed under the terms of the Creative Commons Attribution 4.0 (CC-BY) License. Further distribution of this work is permitted, provided the original work is properly cited. 
model by including the effect of the MHS and thus modeling the impact it has on the spread of dengue within the flats. Numerical simulations and tables are also produced to illustrate our results.

Keywords: Dengue; Auto-Dissemination Trap; Mosquito Home System; Ordinary Differential Equations; Delayed Model; Aedes Aegypti; Aedes Albopictus; Numerical Simulations; Malaysia.

\section{Introduction}

Dengue is a vector-borne disease which is mainly transmitted by two types of Aedes mosquitoes, the main one for transmission is the Aedes Aegypti and the second one is Aedes Albopictus, where Aedes Aegypti mosquitoes are also responsible for the transmission of other diseases such as yellow fever and the zika virus. ${ }^{113}$ Dengue can be a life-threatening disease as infected individuals can develop severe dengue which includes dengue hemorrhagic fever. The World Health Organization has stated that every year there are around 50-100 million dengue infections where at least 100 countries have a dengue epidemic ${ }^{2}$ Malaysia being one of them.

Between 2014-2016, Malaysia had around 330,891 reported dengue cases with around 788 dengue-related deaths with a high incidence rate of 396.4 per 100,000 population in 2015 causing Malaysia to suffer from serious economic and health burdens. In the study carried out by Packierisamy et al., 4 it is estimated that in 2010, it had cost Malaysia around USD $\$ 73.45$ million in dengue-related vector control which was around USD $\$ 2.63$ per capita population. As a result, it is crucial that we find a way to control the spread of dengue in Malaysia. One of the main ways which Malaysia has used to battle against dengue is to use outdoor space spraying commonly known as chemical fogging. However, space spraying is most effective one month after the spraying has taken place and the effect will reduce over time ${ }^{[5}$ In addition, space spraying is a passive approach as spraying will take place only in areas where dengue cases have been reported. One of the main challenges in controlling the spread of dengue is that it is often difficult to find all breeding sites of Aedes mosquitoes. As a result, there is a new method by which we could overcome the problem, namely the Mosquito Home System (MHS) which is an autodissemination trap. The mosquito trap is called an auto-dissemination trap because it contains a solution which the mosquitoes disseminate to other breeding sites. The MHS is a container which contains a special solution that will attract the female Aedes mosquitoes to lay their eggs within the container. Furthermore, any eggs that are laid inside the MHS will get killed off by the solution inside thus preventing those eggs from hatching into adult Aedes mosquitoes. In other words, the MHS will be able to reduce the Aedes mosquito population available to transmit the dengue virus. Before we can conclude on the effectiveness of the MHSs in reducing the number of dengue cases, it is crucial for us to analyze in detail the effect of using the MHS. As a result, in this paper, we will use the well-known classical Ross-Macdonald model ${ }^{6}$ to describe the spread of dengue between humans and 
Aedes mosquitoes in Malaysia. Most importantly, we will also perform mathematical modeling to investigate the impact of the MHSs on reducing the Aedes population and thus reduce the number of dengue cases. In order to improve on the accuracy and the reliability of our analysis, it is important to point out that we will be using the actual experimental field data obtained from deploying the MHSs in three highly urbanized residential condominia known as Ridzuan Court Sunway in Selangor of Malaysia, ${ }^{7}$ where the area is responsible for about $60 \%$ of the total dengue cases in Malaysia.

This paper is arranged as follows: In Sec. 2 we will introduce the classical Ross-Macdonald model as well as the basic reproduction number. In Sec. 3 we will mathematically estimate the number of potential breeding sites of Aedes mosquitoes and perform some basic analysis on the effect of using the MHSs in reducing the number of dengue cases in the trial site. In Sec. 4 we constructed a list of different biting probabilities corresponding to different amounts of time spent outdoors. In Sec. 15. we will perform thorough analysis on the effect of having different levels of MHSs on the number of dengue cases in those three high-rise condominia. Lastly in Sec. 6, we will summarize all our results. Numerical simulations and tables are produced throughout the paper to illustrate our findings.

\section{The Dengue Model and the Basic Reproduction Number}

Let us consider the following delayed SIR model for dengue mentioned in Ref. 8:

$$
\begin{aligned}
\frac{d S_{H}(t)}{d t} & =-a b I_{v}(t) \frac{S_{H}(t)}{N_{H}}-\mu_{H} S_{H}(t)+\mu_{H} N_{H}, \\
\frac{d I_{H}(t)}{d t} & =a b I_{v}(t) \frac{S_{H}(t)}{N_{H}}-\left(\mu_{H}+\gamma\right) I_{H}(t), \\
\frac{d R_{H}(t)}{d t} & =\gamma I_{H}(t)-\mu_{H} R_{H}(t), \\
\frac{d S_{v}(t)}{d t} & =-a c S_{v}(t) \frac{I_{H}(t)}{N_{H}}-\mu_{v} S_{v}(t)+\mu_{v} N_{v}, \\
\frac{d L_{v}(t)}{d t} & =a c S_{v}(t) \frac{I_{H}(t)}{N_{H}}-\mu_{v} L_{v}(t)-a c S_{v}(t-\tau) \frac{I_{H}(t-\tau)}{N_{H}} e^{-\mu_{v} \tau}, \\
\frac{d I_{v}(t)}{d t} & =a c S_{v}(t-\tau) \frac{I_{H}(t-\tau)}{N_{H}} e^{-\mu_{v} \tau}-\mu_{v} I_{v}(t),
\end{aligned}
$$

with initial conditions $S_{H}(0), I_{H}(0)$ and $R_{H}(0)$, where $S_{H}(t), I_{H}(t)$ and $R_{H}(t)$ represent, respectively the susceptible, infected and recovered humans, while $S_{v}(0), L_{v}(0)$ and $I_{v}(0)$ denote the initial conditions for $S_{v}(t), L_{v}(t)$ and $I_{v}(t)$ which represent, respectively the susceptible, latent and infected mosquitoes. Note that $N_{H}=S_{H}+I_{H}+R_{H}$ denotes the total population size for humans and $N_{v}=S_{v}+L_{v}+I_{v}$ represents the total population for Aedes mosquitoes where 
they are both constant. $a$ represents the biting rate for Aedes mosquitoes, $b$ is the dengue transmission probability when an infectious mosquito bites a susceptible human and $c$ is the dengue transmission probability when a susceptible mosquito bites an infected human. $\mu_{H}$ and $\mu_{v}$ are the per capita mortality rates for humans and Aedes mosquitoes, respectively. $\gamma$ is the per capita human recovery rate and $\tau$ is the dengue extrinsic incubation period.

In Eq. (2.1), some of the parameters are easy to estimate. For example $\mu_{H}$ is the reciprocal of the average human lifetime. Similarly $\mu_{v}$ is the reciprocal of the average mosquito lifetime and $\gamma$ is the reciprocal of the infectious period. Other parameters such as $N_{v}$, the total number of mosquitoes, are more difficult to estimate. However, we also have data on the initial phase of the epidemic and can use this to estimate $\lambda$, the initial exponential growth rate of the number of dengue cases, by fitting a function $A e^{\lambda t}$, where $A$ is a constant, to the initial stage of an epidemic. From this $\lambda$ estimate, we can find the basic reproduction number $R_{0}$, and from $R_{0}$, we can estimate $N_{v}$. (Recall that $R_{0}$ represents the expected number of secondary cases that will arise from a single infected person entering a disease-free population at equilibrium. Alternatively, $R_{0}$ can be thought of as the expected number of secondary cases that will arise from a single infected mosquito entering a diseasefree population at equilibrium.)

The Appendix of Ref. 9 gives a method to estimate $R_{0}$ for a very similar model from the easily estimated parameters $\lambda, \mu_{H}, \mu_{v}, \tau$ and $\gamma$, by looking at the initial exponential phase of the number of dengue cases. We shall use this method to estimate $R_{0}$ for our model (see Eq. (2.4)). Then we shall give an alternative expression for $R_{0}$, Eq. (2.5), derived from the differential equation model, Eqs. (2.1), and use it to estimate $m$, the number of Aedes mosquitoes per human, and hence to estimate $N_{v}$, the total number of mosquitoes.

We apply the estimation method in the Appendix of Ref. 9 to our model. Our model is similar to the model discussed in Ref. 9 but additionally includes both the per capita human death rate, $\mu_{H}$, and the transmission probability per bite from an infected human to a susceptible mosquito, $c$. Moreover there is a mistake in the $R_{0}$ calculation in Ref. 9] In the second equation of each of Eqs. (9) and (10) in Ref. 9, the argument of $i_{H}$, which is not explicitly stated, should be $t-\tau$ (not $t$ ). Following the method outlined in Ref. 9 gives

$$
\begin{aligned}
R_{0} & =\left(1+\frac{\lambda^{2}+\lambda(\mu+\gamma)}{\mu \gamma}\right) e^{\lambda \tau}, \\
\text { not } \quad R_{0} & =\left(1+\frac{\lambda^{2}+\lambda(\mu+\gamma)}{\mu \gamma}\right) .
\end{aligned}
$$

So the $R_{0}$ value stated in Ref. 9 is 2.3 but it should be (2.2). Note that $\mu$ in Ref. 9 corresponds to $\mu_{v}$ in our model. 
Applying the same method to our model, it is straightforward to obtain

$$
R_{0}=\frac{\left(\lambda+\mu_{v}\right)\left(\lambda+\mu_{H}+\gamma\right) e^{\lambda \tau}}{\left(\mu_{H}+\gamma\right) \mu_{v}} .
$$

In order to calculate the basic reproduction number, we need to know the parameter values. According to the World Bank data, $\frac{10}{10}$ the life expectancy in Malaysia in 2015 was 75 years and thus $\mu_{H}=1 /(75 \times 365)$ per day. The average lifespan for an Aedes mosquito is around 2 weeks according to the Singapore National Environment Agency $\underline{11}$ and thus $\mu_{v}=1 / 14$ per day. The human recovery rate is $\gamma=1 / 7$ per day and the extrinsic incubation period is around 8 days 12

By using the dengue data available in Ref. 13 which contains the number of dengue cases in Malaysia in 2013, and by using numerical simulation in $\mathrm{R}$, we have plotted an exponential curve fitted against the real data to estimate $\lambda$, which recall represents the initial growth rate of the dengue epidemic. In this case, we have that $\lambda=0.0053 /$ day. By substituting all the required parameter values into Eq. (2.4), we have that $R_{0}=1.162$.

One of the main difficulties when modeling the spread of dengue is that it is extremely difficult to know the number of Aedes mosquitoes that are in the country. However, we can estimate this using an alternative definition of the basic reproduction number which is expressed in terms of $m$, where $m=N_{v} / N_{H}$ is the number of Aedes mosquitoes per one human. The basic reproduction number derived from the differential equation model (2.1) is given as follows:

$$
R_{0}=\frac{m a^{2} b c e^{-\mu_{v} \tau}}{\mu_{v}\left(\mu_{H}+\gamma\right)},
$$

where $a=0.25$ is the Aedes mosquitoes daily biting rate, $b=0.75$ and $c=0.37514$ Similarly by substituting the parameter values and setting $R_{0}=1.162$, it is clear that $m=1.195$. As a result, the number of Aedes mosquitoes can be estimated using $N_{v}=m N_{H}$. Note that both Eqs. (2.4) and (2.5) represent the basic reproduction number, but they are derived in different ways.

In the next section, we will focus on analyzing the effect of the MHS on the spread of dengue within the three blocks of flats in Selangor where an 11 months MHS trial has been carried out.

\section{The Effect of the MHS on the Flats}

Throughout this paper, unless stated otherwise, all figures will be rounded to three decimal places with the unit of time in weeks.

In order to analyze the effect of the MHS on the spread of dengue within the flats, we will modify the delayed dengue model (2.1) into a more complicated one where we are now working with 12 differential equations, where six of them describe the disease dynamics in Kuala Lumpur (KL) while the other six represent the dynamics 
of the disease within the flats with the effect of the MHS. Let us define the new model as follows:

$$
\begin{aligned}
& \frac{d S_{H_{1}}(t)}{d t}=-a b I_{v_{1}}(t) \frac{S_{H_{1}}(t)}{N_{H_{1}}}-\mu_{H} S_{H_{1}}(t)+\mu_{H} N_{H_{1}}, \\
& \frac{d I_{H_{1}}(t)}{d t}=a b I_{v_{1}}(t) \frac{S_{H_{1}}(t)}{N_{H_{1}}}-\left(\mu_{H}+\gamma\right) I_{H_{1}}(t) \\
& \frac{d R_{H_{1}}(t)}{d t}=\gamma I_{H_{1}}(t)-\mu_{H} R_{H_{1}}(t) \\
& \frac{d S_{v_{1}}(t)}{d t}=-a c S_{v_{1}}(t) \frac{I_{H_{1}}(t)}{N_{H_{1}}}-\mu_{v} S_{v_{1}}(t)+\mu_{v} N_{v_{1}} \\
& \frac{d L_{v_{1}}(t)}{d t}=a c S_{v_{1}}(t) \frac{I_{H_{1}}(t)}{N_{H_{1}}}-\mu_{v} L_{v_{1}}(t)-a c S_{v_{1}}(t-\tau) \frac{I_{H_{1}}(t-\tau)}{N_{H_{1}}} e^{-\mu_{v} \tau}, \\
& \frac{d I_{v_{1}}(t)}{d t}=a c S_{v_{1}}(t-\tau) \frac{I_{H_{1}}(t-\tau)}{N_{H_{1}}} e^{-\mu_{v} \tau}-\mu_{v} I_{v_{1}}(t), \\
& \frac{d S_{H_{2}}(t)}{d t}=-a b\left(P \frac{I_{v_{1}}(t)}{N_{H_{1}}}+(1-P) \frac{I_{v_{2}}(t)}{N_{H_{2}}}\right) S_{H_{2}}(t)-\mu_{H} S_{H_{2}}(t)+\mu_{H} N_{H_{2}}, \\
& \frac{d I_{H_{2}}(t)}{d t}=a b\left(P \frac{I_{v_{1}}(t)}{N_{H_{1}}}+(1-P) \frac{I_{v_{2}}(t)}{N_{H_{2}}}\right) S_{H_{2}}(t)-\left(\mu_{H}+\gamma\right) I_{H_{2}}(t), \\
& \frac{d R_{H_{2}}(t)}{d t}=\gamma I_{H_{2}}(t)-\mu_{H} R_{H_{2}}(t), \\
& \frac{d S_{v_{2}}(t)}{d t}=-a c S_{v_{2}}(t) \frac{I_{H_{2}}(t)}{N_{H_{2}}}-\mu_{v} S_{v_{2}}(t)+\mu_{v} N_{v_{2}}\left(1-P^{*}\right), \\
& \frac{d L_{v_{2}}(t)}{d t}=a c S_{v_{2}}(t) \frac{I_{H_{2}}(t)}{N_{H_{2}}}-\mu_{v} L_{v_{2}}(t)-a c S_{v_{2}}(t-\tau) \frac{I_{H_{2}}(t-\tau)}{N_{H_{2}}} e^{-\mu_{v} \tau}, \\
& \frac{d I_{v_{2}}(t)}{d t}=a c S_{v_{2}}(t-\tau) \frac{I_{H_{2}}(t-\tau)}{N_{H_{2}}} e^{-\mu_{v} \tau}-\mu_{v} I_{v_{2}}(t),
\end{aligned}
$$

where all the parameters are defined as before and Eq. 3.1 represent the transmission of dengue between humans and Aedes mosquitoes in KL, while Eq. (3.2) represent the transmission of dengue within the three blocks of flats. The parameter $P$ denotes the fraction of time in which an average person resident in the flats stays in KL, while $1-P$ represents the fraction of time an average person stays inside the flats. $P^{*}$ represents the total reduction in the proportion of Aedes mosquitoes population as a result of using the MHS inside the three blocks of flats.

Before we solve the 12 differential equations using $\mathrm{R}$, it is useful to estimate the potential number of mosquito sites that are in the vicinity of the flats, in order to determine the appropriate number of MHSs that would be needed to achieve a substantial effect in reducing the number of dengue cases. 
From a previous trial carried out in the Jacob-Ballas Childrens' Park at Singapore Botanic Gardens, $\frac{15}{15}$ we know that there were 46 MHSs and 46 Gravitraps used to collect Aedes eggs. A Gravitrap is a simple cylindrical black container with sticky surface which allows the female Aedes mosquitoes to lay their eggs. ${ }^{16}$ Note that the Gravitraps used do not contain the special solution which lures the female Aedes. The total number of eggs collected by the MHSs were approximately 12 times more than the ones collected by the Gravitraps. An increase is to be expected as the MHS has a special chemical within the trap that naturally attracts Aedes mosquitoes to lay eggs within it.

In the absence of any information to the contrary, we assume that without the MHSs the mosquitoes choose one of the available breeding sites at random to lay their eggs. However, we know that at the Jacob-Ballas Childrens' Park in the Singapore Botanic Gardens trial, the MHSs were approximately 12 times as attractive to mosquitoes as the Gravitraps, which are the only other available breeding sites on which we have information. Hence we assume that the MHSs are 12 times more attractive than other potential breeding sites. Let us assume that there are $x$ MHSs being deployed, $o$ ovitraps and $y$ hidden breeding sites. Then the probability that an egg is laid in one of the $x$ MHSs is

$$
\frac{12 x}{12 x+o+y} \text {. }
$$

As there are $k$ eggs altogether, then the total expected number of eggs laid in the MHSs is

$$
k \times \frac{12 x}{12 x+o+y}=\frac{12 k x}{12 x+o+y} .
$$

So this is the expected number of eggs collected by the MHSs within the three blocks of flats. This will be used later on in Eq. (3.6) to estimate the number of breeding sites.

From Fig. 6]in Ref. 7], we have that the mean number of eggs per paper collected in all three blocks of flats using the MHS has decreased to around $70.65 \%$ of its value before introduction after extra MHSs were introduced into block B thus increasing the total number of MHSs deployed in the flats from 344 to 552 . Each of the MHSs contains a piece of tissue paper which dips into the solution and the mosquitoes lay their eggs on it. Therefore the mean number of hidden breeding sites can be estimated, as the mean number of eggs per paper can be expressed as follows:

$$
\frac{1}{x} \times \frac{12 k x}{12 x+o+y}=\frac{12 k}{12 x+o+y} .
$$

As a result,

$$
\frac{0.7065 \times 12 k}{12 \times 344+356+y}=\frac{12 k}{12 \times 552+356+y},
$$

as $o=356$. By re-arranging Eq. (3.6) and solving for $y$, which represents the number of hidden sites, we have that $y=1524$. This means that on average each block of 
flats has around 505 breeding sites of Aedes mosquitoes. This possibly explains why initially having 344 MHSs in all blocks of flats, in other words around 114 MHSs in each block has very little effect on reducing the Ovitrap Index as it is relatively low compared to our estimated number of Aedes sites. However as soon as the number of MHSs in block B was increased from the initial 116 to 324, we see a drastic decrease in Ovitrap Index as obviously the number of MHSs used in block B is more than half of the potential breeding sites associated with that block, assuming that the breeding sites are equally distributed among the blocks.

We will now solve the differential equations given by Eqs. 3.1 and (3.2) and produce some numerical simulations to demonstrate graphically what happened to the dynamics of dengue.

Example 3.1. Let us define all the parameter values the same as before but changing the Aedes mosquitoes' daily biting rate to 0.20 and thus $m$ would become 1.867 . According to the data given in Ref.17, the total population in KL in 2013 is around $1,720,000$ and thus the estimated number of Aedes mosquitoes using the relation that $N_{v}=m N_{H}$ is around 2,055,632. Each block of flats has 10 units of houses per floor and thus in total there will be 800 units of houses in all three blocks of flats. By assuming that each house has an average of four residents, then $N_{H_{2}}=3,200$ and $N_{v_{2}}=3,824.432$.

The incidence rate in KL in 2013 was 146.7 per year per 100,000 population. 18 Typically, dengue cases have a low ratio of symptomatic to asymptomatic cases of 1 to 4, respectively and thus we will increase the incidence rate by 5 times to reflect this situation. In addition, from 1995 to 2012, the number of dengue cases in Malaysia was around 505,264 (without taking into consideration the asymptomatic cases). From previous data on the number of reported dengue cases in different states in Malaysia, KL is responsible for around $3-10 \%$ of those cases. Therefore, by assuming that KL is responsible for around $5 \%$ of those dengue cases and assuming that everyone was infected by the same serotype, we can estimate the number of recovered individuals in 2013. The unit of time, unless stated otherwise, is in weeks.

Let us define the initial values of Eqs. (3.1) and (3.2) to be

$$
\begin{array}{cl}
S_{H_{1}}(0)=1,593,441, & I_{H_{1}}(0)=242.619, \quad R_{H_{1}}(0)=126,316, \\
S_{v_{1}}(0)=3,211,447, & L_{v_{1}}(0)=207.042, \quad I_{v_{1}}(0)=268.608, \\
S_{H_{2}}(0)=2,964.542, & I_{H_{2}}(0)=0.451, \quad R_{H_{2}}(0)=235.007, \\
S_{v_{2}}(0)=5,974.785, & L_{v_{2}}(0)=0.385, \quad I_{v_{2}}(0)=0.4997,
\end{array}
$$

where we have set the initial values within the flats to reflect the distribution of the initial values in KL. Note that the initial conditions for the number of susceptible, latent and infected mosquitoes in KL are obtained using the initial values of $I_{H_{1}}(0)$ and $N_{H_{1}}$ and substituting them into the differential equations for $\frac{d S_{v_{1}}(t)}{d t}, \frac{d L_{v_{1}}(t)}{d t}$ and $\frac{d I_{v_{1}}(t)}{d t}$. 
It is known that the Aedes mosquito bites more often at dawn and dusk, 19 therefore people are most likely to stay indoors during those periods, and thus it is reasonable to assume that $P$ is relatively small, say $P=0.10$, where recall that $P$ is the proportion of time that an average person resident in the flats is in KL.

From the Singapore Park data, $\frac{15}{15}$ we know that during the two months trial, the total reduction in the Aedes population was around 0.5889 , and thus by using this as a rough guide, we will set $P^{*}=0.5889$.

The numerical simulations for the spread of dengue in KL are shown in Fig. [1] including the incidence cases and the total number of cases over a period of three years.

The numerical simulation for the number of incidence cases in weeks and the total number of dengue cases within the blocks of flats is given in Fig. 2. The red lines shown in both figures represent the effect of using the MHSs in the flats and thus reducing the Aedes mosquitoes population by a factor of 0.5889 . The black line represents the effect of having no MHSs present within the blocks of flats. From Fig. 2. it is clear that the MHSs have effectively reduced the number of dengue incidences. This is also confirmed in simulation (b) of Fig. 2 where we have seen
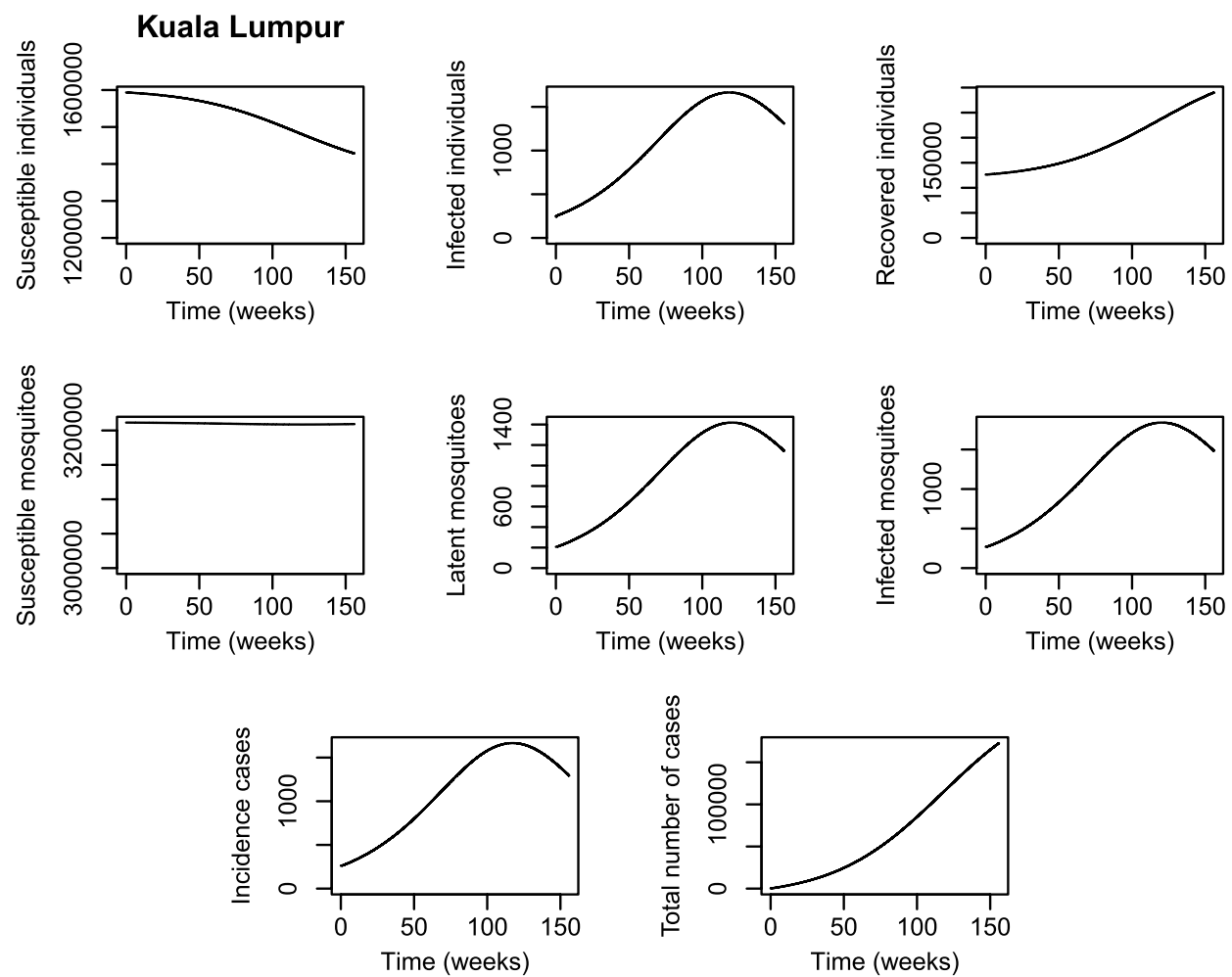

Fig. 1. Numerical simulation for our solution to Eq. 3.1 over a period of three years. 


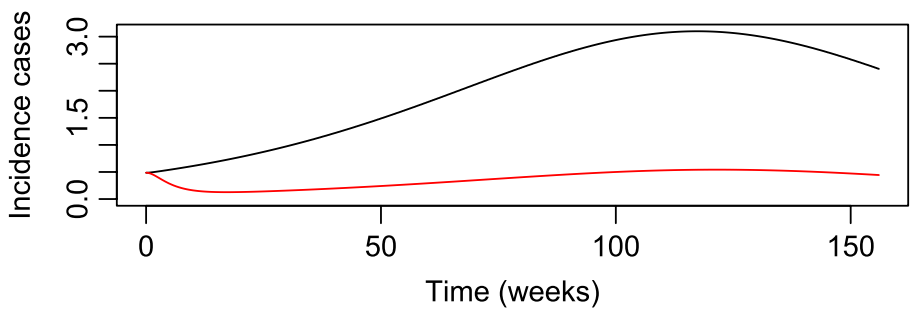

(a)



(b)

Fig. 2. Numerical simulation for (a) number of incidence cases per week and (b) total number of cases within the blocks of flats with $P=0.10$ where the black line and red line represent $P^{*}=0$ and $P^{*}=0.5889$ respectively over a period of three years.

the total number of cases over a period of three years have reduced drastically as a result of using the MHSs within the blocks of flats. Note that we have also produced the numerical simulation for the number of infected individuals within the blocks of flats, however the simulation has the same qualitative behavior as the number of dengue incidence cases per week shown. Therefore we have decided to omit the simulation in Fig. 2. This is the case throughout the paper for other similar simulations.

The dynamical behavior for the Aedes mosquitoes within the flats is illustrated in Fig. 3, where the red line represents the effect from the MHSs and the black line represents the effect when we have no MHSs. Again the effect is clear as shown in the simulations. Note also that the numerical simulation for the number of latent Aedes mosquitoes was also produced, however the simulation has the same qualitative behavior as the number of infected Aedes mosquitoes shown. Therefore we have omitted the latent mosquitoes figure in Fig. 3 This is the case throughout the paper for other similar simulations.

From Table 1, we can see clearly that by introducing the MHSs in the three blocks of flats, not only did the mean number of infected Aedes mosquitoes reduce by a high proportion of $87.87 \%$, the mean number of infected individuals has also decreased dramatically by about $78.04 \%$. In addition, the mean number of dengue incidence cases has reduced by $78.94 \%$. These results are very promising, as they 

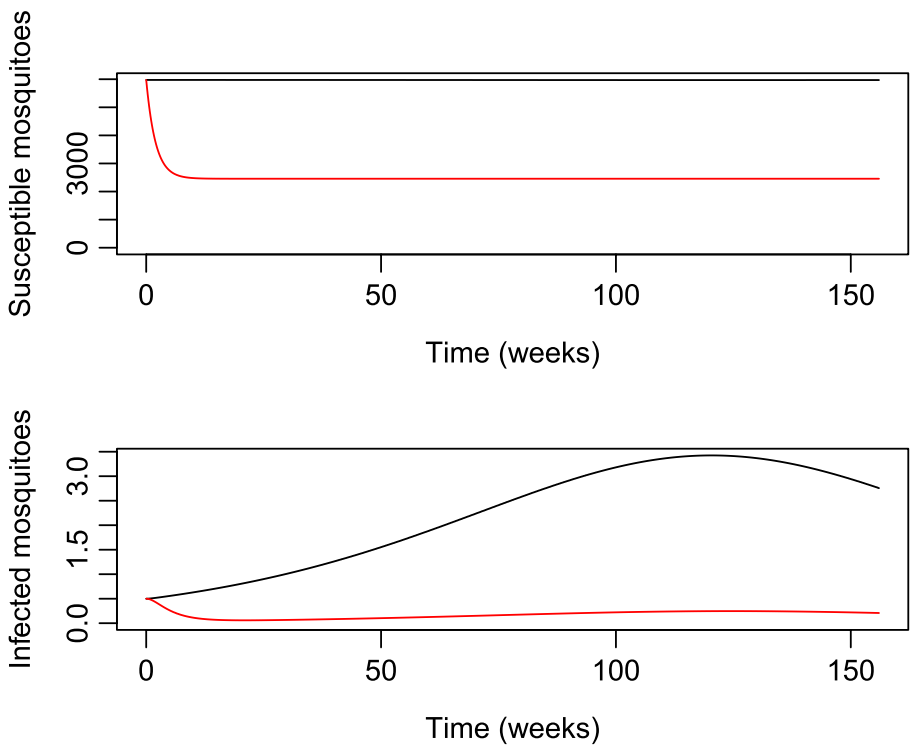

Fig. 3. Dynamical behavior of Aedes mosquitoes within the blocks of flats with $P=0.10$ and the black line and red line represent $P^{*}=0$ and $P^{*}=0.5889$ respectively over a period of three years.

Table 1. Mean values for variables in flats with and without the MHSs to 3 d.p after one year where $P=0.10$.

\begin{tabular}{|c|c|c|c|}
\hline Variable values & $P^{*}=0$ & $P^{*}=0.5889$ & Percentage change \\
\hline $\begin{array}{l}\text { Susceptible individuals } \\
S_{H_{2}}(t) \text { in flats }\end{array}$ & $2,946.55$ & $2,960.778$ & $\begin{array}{c}\text { Increased by } \\
0.48 \%\end{array}$ \\
\hline $\begin{array}{l}\text { Infected individuals } \\
I_{H_{2}}(t) \text { in flats }\end{array}$ & 0.912 & 0.200 & $\begin{array}{c}\text { Decreased by } \\
78.04 \%\end{array}$ \\
\hline $\begin{array}{l}\text { Recovered individuals } \\
R_{H_{2}}(t) \text { in flats }\end{array}$ & 252.538 & 239.022 & $\begin{array}{c}\text { Decreased by } \\
5.35 \%\end{array}$ \\
\hline $\begin{array}{l}\text { Susceptible Aedes mosquitoes } \\
S_{v_{2}}(t) \text { in flats }\end{array}$ & $5,973.959$ & $2,591.714$ & $\begin{array}{c}\text { Decreased by } \\
56.62 \%\end{array}$ \\
\hline $\begin{array}{l}\text { Infected Aedes mosquitoes } \\
I_{v_{2}}(t) \text { in flats }\end{array}$ & 0.966 & 0.117 & $\begin{array}{c}\text { Decreased by } \\
87.87 \%\end{array}$ \\
\hline $\begin{array}{l}\text { Latent Aedes mosquitoes } \\
L_{v_{2}}(t) \text { in flats }\end{array}$ & 0.745 & 0.09 & $\begin{array}{l}\text { Decreased by } \\
\quad 87.92 \%\end{array}$ \\
\hline Incidence cases & 0.933 & 0.196 & $\begin{array}{c}\text { Decreased by } \\
78.94 \%\end{array}$ \\
\hline Total cases & 48.984 & 10.696 & $\begin{array}{c}\text { Decreased by } \\
78.16 \%\end{array}$ \\
\hline
\end{tabular}

highlight the fact that the MHSs have effectively reduced the number of dengue cases within the flats by reducing the number of Aedes mosquitoes. Furthermore, the impact ratio which can be calculated using

$$
1-\frac{\text { cases after }}{\text { cases before }}
$$


Table 2. Endemic equilibrium values for variables in flats with and without the MHSs to 3 d.p where $P=0.10$.

\begin{tabular}{lccc}
\hline Variable values & $P^{*}=0$ & $P^{*}=0.5889$ & Percentage change \\
\hline $\begin{array}{l}\text { Susceptible individuals } \\
\text { in flats } S_{H_{2}}(t)\end{array}$ & $2,753.155$ & $3,113.127$ & $\begin{array}{c}\text { Increased by } \\
13.07 \%\end{array}$ \\
$\begin{array}{l}\text { Infected individuals } \\
\text { in flats } I_{H_{2}}(t)\end{array}$ & 0.115 & 0.022 & $\begin{array}{c}\text { Decreased by } \\
80.87 \%\end{array}$ \\
$\begin{array}{l}\text { Recovered individuals } \\
\text { in flats } R_{H_{2}}(t)\end{array}$ & 446.730 & 86.850 & $\begin{array}{c}\text { Decreased by } \\
80.56 \%\end{array}$ \\
$\begin{array}{l}\text { Susceptible Aedes mosquitoes } \\
\text { in flats } S_{v_{2}}(t)\end{array}$ & $5,975.446$ & $2,456.580$ & $\begin{array}{c}\text { Decreased by } \\
58.89 \%\end{array}$ \\
$\begin{array}{l}\text { Infected } A e d e s \text { mosquitoes } \\
\text { in flats } I_{v_{2}}(t)\end{array}$ & 0.127 & 0.010 & $\begin{array}{c}\text { Decreased by } \\
92.13 \%\end{array}$ \\
$\begin{array}{l}\text { Latent } A e d e s \text { mosquitoes } \\
\text { in flats } L_{v_{2}}(t)\end{array}$ & 0.098 & 0.008 & $\begin{array}{c}\text { Decreased by } \\
92.84 \%\end{array}$ \\
$\begin{array}{l}\text { Incidence cases } \\
\text { Total cases (per year) }\end{array}$ & 0.115 & 0.022 & $\begin{array}{c}\text { Decreased by } \\
80.87 \%\end{array}$ \\
& 5.958 & 1.158 & $\begin{array}{c}\text { Decreased by } \\
80.56 \%\end{array}$ \\
\hline
\end{tabular}

shows that the impact of introducing the MHSs in the three blocks of flats has a value of 0.7816 .

Note that the mean number of susceptible individuals within the flats have increased when we have the MHSs, this is possibly due to the fact that the rate of infection is slowing down as there are now less Aedes mosquitoes to spread the dengue virus.

The corresponding endemic equilibrium values for the number of infected individuals, incidence cases and total dengue cases within the blocks of flats with and without using the MHSs are shown in Table 2. The results shown in Table 2 further highlight the impact of using the MHSs.

Note that the cumulative number of dengue cases in KL in 2013 over a period of 40 weeks was 8420 (after multiplying $1684^{20}$ by 5 to take into consideration asymptomatic cases). From our numerical simulations given in Example [3.1, we have that the total number of cases after 40 weeks is around 17,323.918. To further investigate this scenario, we run the simulations for a longer period of time until the end of 2015. The cumulative number of dengue cases in Malaysia from 2013 to 2015 was 272,880 . By making the same assumption that KL was responsible for $5 \%$ of the overall dengue cases in Malaysia in 2015, then from 2013 to 2015, KL had around 68,220 dengue cases (after multiplying 13,644 by 5 to take into consideration asymptomatic cases). From our numerical simulation, at the end of 2015, we expect to have $91,548.72$ which is around 1.34 times more than the actual number of cases.

Although it seems that our numerical simulation has over-predicted the actual dengue data by around 1.34 times, it is important to note that this could be due to the symptomatic to asymptomatic cases ratio that we chose in Example 3.1 Note 
that the estimation of this ratio varies greatly. For example, Ref. 21 used the ratio of 1:3.9 for the symptomatic to asymptomatic cases ratio which is very close to the 1:4 ratio that we used in the previous example, while in Ref. 22, Wilder-Smith et al. have chosen the symptomatic to asymptomatic cases ratio for the dengue cases in Singapore to range from 1:2.1 to $1: 10$. As a result, it would be interesting to know what would happen if we choose a different symptomatic to asymptomatic cases ratio than the one in Example 3.1. The results are shown in the following example.

Note that from the official report from the Ministry of Health in Malaysia, Ref. 20 the cumulative dengue cases in KL in 2013 over a period of 40 weeks was 1684 , but by using the incidence rate in KL in 2013, namely 146.7 per $100,000, \frac{18}{18}$ the number of reported cases should be around 1940.90. The difference could be due to not having the exact number of population of KL.

Example 3.2 (1:9 ratio). By choosing the symptomatic to asymptomatic cases ratio for dengue to be 1:9, the new initial values of Eqs. (3.1) and (3.2) are

$$
\begin{aligned}
& S_{H_{1}}(0)=1,466,883, \quad I_{H_{1}}(0)=485.239, \quad R_{H_{1}}(0)=252,632, \\
& S_{v_{1}}(0)=3,210,972, \quad L_{v_{1}}(0)=414.022, \quad I_{v_{1}}(0)=537.136, \\
& S_{H_{2}}(0)=2,729.084, \quad I_{H_{2}}(0)=0.903, \quad R_{H_{2}}(0)=470.013, \\
& S_{v_{2}}(0)=5,973.901, \quad L_{v_{2}}(0)=0.770, \quad I_{v_{2}}(0)=0.999,
\end{aligned}
$$

where all the parameter values are defined as before.

By carrying out the same numerical simulations as in Example 3.1 we have that the cumulative number of cases in KL over the first 40 weeks in 2013 was 17,959.94. The actual cumulative number of dengue cases in KL over the same period was 16,840 (after multiplying $1,684^{20}$ by 10 to take asymptomatic cases into consideration). Similarly to before, we continue the simulation for a longer period of time until the end of 2015 to get the total number of cases in KL to be 37,897 . The actual dengue cases data at the end of 2015 was 136,440 (after multiplying 13,644 by 10 to take into consideration asymptomatic cases). As a result, for this ratio, our estimated total number of dengue cases is around 3.6 times less than the actual dengue cases. This could be due to the fact that the dengue incidence rate in 2015 was about 2.72 times more than the one in 2013.

By comparing the results from two different symptomatic to asymptomatic ratios, it seems that a 1:4 ratio gives a more accurate result when comparing the estimated data with the actual dengue data given in Ref. 20. Therefore, for the rest of the paper, we will continue the analysis by assuming that the symptomatic to asymptomatic ratio is $1: 4$.

Note that the absolute numbers of infected individuals and infected and latent mosquitoes in the flats are very small (less than one) as shown in Tables 1 and 2 . Hence at these levels of small numbers of infected individuals and mosquitoes, a 
deterministic model strictly speaking should not be used and a stochastic model would be preferable. Nonetheless, the deterministic model can still give insight into the behavior of the system, particularly when comparing the number of cases in the flats with and without the MHSs or later (in Sec. 4) when comparing the equilibrium values with different values of $P$.

Note also that the number of dengue cases in KL will be large so for that part of the model a deterministic model is appropriate. As the disease is endemic in KL, there is a constant source of infection from outside the flats. So the disease will not die out in the flats.

\section{Proportion of Time Outdoors}

Recall from Sec. 3 that $P$ denotes the fraction of time in which an average person resident in the flats stays in $\mathrm{KL}$ and $P^{*}$ represents the total reduction in the proportion of Aedes mosquitoes as a result of the MHSs. From the simulations carried out, it is clear that the two parameter values $P$ and $P^{*}$ play an important role in controlling the spread of dengue within the flats. Therefore in the next two sections, we will focus on these two parameter values.

First of all, we will look at the effect of having different $P$ values. In this section, we will use the biting facts on Aedes mosquitoes and interpret $P$ in a slightly different way where $P$ represents the proportion of bites a person will get if going outdoors in a given time interval and thus $1-P$ represents the proportion of bites if a person remains inside. The values of $P$ will thus vary depending on the time the person decides to go out. From the biting facts data available collected in April 2007, the three time slots which have the highest Aedes' biting activity are between 6-6.45 am, 5-5.45 pm and 6-6.45 pm as mosquitoes are more likely to bite at dusk and dawn.

From Table 3, we have five different time slots in which a person decides to leave the house and be in KL and remain indoors with their corresponding $P$ and $1-P$ values, respectively.

By keeping the parameter values the same as in Example 3.1 where $P^{*}=0.5889$ and solving the differential equations for different $P$ values, we have the results shown in Table 4 at the end of one year. From Table 4 , it is clear that as $P$ decreases, the number of incidence cases, the total number of dengue cases, infected individuals

Table 3. Proportions of Aedes bites according to biting activities at different time intervals.

\begin{tabular}{lcc}
\hline Outdoor Time & $P$ (outdoor) & $1-P$ (indoor) \\
\hline 6 am-7.45 pm & 0.6610 & 0.3390 \\
$6 \mathrm{am}-6.45 \mathrm{pm}$ & 0.6210 & 0.3790 \\
$9 \mathrm{am}-7.45 \mathrm{pm}$ & 0.4770 & 0.5230 \\
$8 \mathrm{am}-5.45 \mathrm{pm}$ & 0.3190 & 0.6810 \\
$9 \mathrm{am}-4.45 \mathrm{pm}$ & 0.1280 & 0.8720 \\
\hline
\end{tabular}


Table 4. Mean values for variables in flats for various values for $P$ with $P^{*}=0.5889$ to 3 d.p after one year.

\begin{tabular}{lrrrrr}
\hline Variable values & $P=0.6610$ & $P=0.6210$ & $P=0.4770$ & $P=0.3190$ & $P=0.1280$ \\
\hline $\begin{array}{l}\text { Susceptible individuals } \\
\text { in flats }\end{array}$ & $2,950.584$ & $2,951.141$ & $2,953.324$ & $2,956.094$ & $2,960.113$ \\
$\begin{array}{l}\text { Infected individuals in } \\
\quad \text { flats }\end{array}$ & 0.714 & 0.686 & 0.578 & 0.439 & 0.234 \\
$\begin{array}{l}\text { Recovered individuals } \\
\quad \text { in flats }\end{array}$ & 248.702 & 248.173 & 246.098 & 243.467 & 239.652 \\
$\begin{array}{l}\text { Susceptible Aedes } \\
\quad \text { mosquitoes in flats }\end{array}$ & $2,591.326$ & $2,591.347$ & $2,591.429$ & $2,591.534$ & $2,591.688$ \\
$\begin{array}{l}\text { Infected Aedes } \\
\text { mosquitoes in flats }\end{array}$ & 0.336 & 0.324 & 0.278 & 0.219 & 0.132 \\
$\begin{array}{l}\text { Latent Aedes } \\
\quad \text { mosquitoes in flats }\end{array}$ & 0.259 & 0.250 & 0.214 & 0.168 & 0.101 \\
$\begin{array}{l}\text { Incidence cases } \\
\text { Total cases in flats }\end{array}$ & 0.728 & 0.699 & 0.587 & 0.439 & 0.232 \\
\hline & 38.331 & 36.848 & 31.010 & 23.533 & 12.532 \\
\hline
\end{tabular}

and infected Aedes mosquitoes in flats also decrease accordingly. A smaller $P$ value means that an individual is outside in KL at a time when Aedes mosquitoes are not as active. As a result, it is reasonable to notice a decrease in incidence rate and infected cases as the chance of a susceptible individual bringing in new infection into the flat will be relatively lower compared to a higher $P$ value.

The corresponding endemic equilibrium values for when $P^{*}=0.5889$ for different values of $P$ are shown in Table 5. We can see that as $P$ decreases, the endemic equilibrium values for the total number of dengue cases within the flats also decrease.

Table 5. Endemic equilibrium values for variables in flats with different $P$ values where $P^{*}=0.5889$ to 3 d.p.

\begin{tabular}{lrrrrr}
\hline Variable values & $P=0.6610$ & $P=0.6210$ & $P=0.4770$ & $P=0.3190$ & $P=0.1280$ \\
\hline $\begin{array}{l}\text { Susceptible individuals } \\
\quad \text { in flats }\end{array}$ & $2,843.632$ & $2,856.567$ & $2,908.634$ & $2,978.761$ & $3,092.447$ \\
$\begin{array}{l}\text { Infected individuals in } \\
\quad \text { flats }\end{array}$ & 0.091 & 0.088 & 0.074 & 0.057 & 0.028 \\
$\begin{array}{l}\text { Recovered individuals } \\
\quad \text { in flats }\end{array}$ & 356.277 & 343.345 & 291.291 & 221.182 & 107.526 \\
$\begin{array}{l}\text { Susceptible Aedes } \\
\quad \text { mosquitoes in flats }\end{array}$ & $2,456.524$ & $2,456.527$ & $2,456.538$ & $2,456.552$ & $2,456.576$ \\
$\begin{array}{l}\text { Infected Aedes } \\
\text { mosquitoes in flats }\end{array}$ & 0.042 & 0.040 & 0.034 & 0.026 & 0.013 \\
$\begin{array}{l}\text { Latent Aedes } \\
\quad \text { mosquitoes in flats }\end{array}$ & 0.032 & 0.031 & 0.026 & 0.020 & 0.010 \\
$\begin{array}{l}\text { Incidence cases } \\
\text { Total cases (per year) }\end{array}$ & 0.091 & 0.088 & 0.075 & 0.057 & 0.028 \\
\hline
\end{tabular}




\section{Different Numbers of MHSs Within the Flats}

In this section, we will focus on analyzing the effect of having different number of MHSs on the spread of dengue, in particular we will look at three different cases, namely

(1) No MHSs within the flats,

(2) 344 MHSs and 356 ovitraps within the flats,

(3) 552 MHSs and 356 ovitraps within the flats.

Recall that $P^{*}$ represents the total reduction in the proportion of Aedes mosquitoes population as a result of using the MHS inside the three blocks of flats. Therefore it can be expressed using the following equation:

$$
P^{*}=\frac{12 x}{12 x+o+y},
$$

where $x, o$ and $y$ are defined as before. In this case $o=356$ and $y=1,524$. By using Eq. (5.1) and substituting all the values for $x, o$ and $y$, we will be able to find the corresponding $P^{*}$ in each case. The $P^{*}$ values for each case are $0,0.6871$ and 0.7789 , respectively.

In the next section, we will show some simulations produced in $\mathrm{R}$ for each case. Note that we will focus on two different $P$ values mentioned in Table 4, namely $P=0.1280$ and $P=0.6610$ as these two are the two extreme $P$ values. Unless stated otherwise, the unit of time is in weeks.

\section{1. $P=0.1280$}

Let us recall again that

(1) $P^{*}=0$, no MHSs,

(2) $P^{*}=0.6871$, there are 344 MHSs and 356 ovitraps within the flats,

(3) $P^{*}=0.7789$, there are $552 \mathrm{MHSs}$ and 356 ovitraps within the flats.

The results for number of incidence cases and the total number of dengue cases within the flats are shown in Fig. 4 The number of susceptible and infected mosquitoes within the flats are shown in Fig. [5. From Fig. 5 we can see that by introducing MHSs in the blocks of flats, the number of susceptible and infected

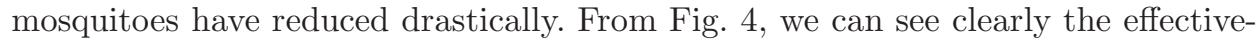
ness of having MHSs within the blocks of flats by seeing a huge reduction in the number of incidence cases as well as the total number of dengue cases. The $P^{*}$ value that performs the best by having the lowest number of infected individuals and number of incidence cases is when we have 552 MHSs inside the flats, namely when $P^{*}=0.7789$ as expected. 


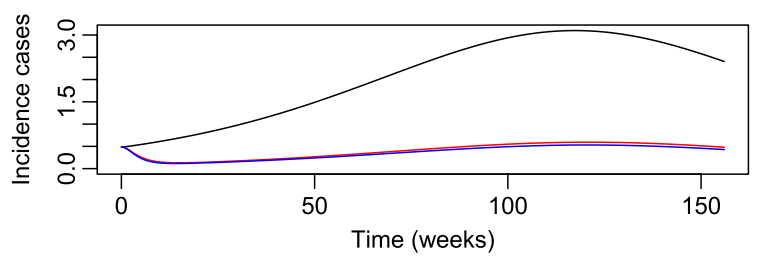

(a)

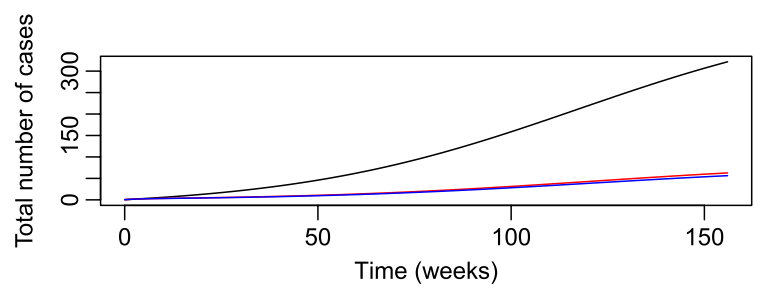

(b)

Fig. 4. Numerical simulations for (a) incidence cases (in weeks) and (b) total number of dengue cases within the flats for $P=0.1280$ where the black line, red line and blue line represent $P^{*}=$ $0, P^{*}=0.6871$ and $P^{*}=0.7789$ respectively over a period of three years.
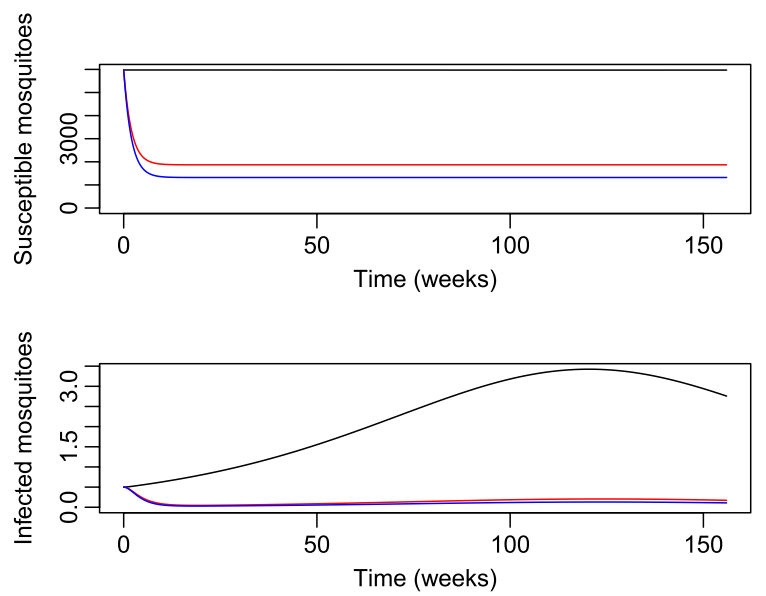

Fig. 5. Numerical simulation for the dynamical behavior of Aedes mosquitoes within the flats for $P=0.1280$ where the black line, red line and blue line represent $P^{*}=0, P^{*}=0.6871$ and $P^{*}=0.7789$ respectively over a period of three years.

\section{2. $P=0.6610$}

Similarly, we will carry out the same procedure for when $P=0.6610$. The results for number of incidence cases and the total number of dengue cases within the flats are shown in Fig. 6. The number of susceptible and infected mosquitoes within the flats are shown in Fig. 7 Similarly, from Fig. 7, we can see that by introducing the 


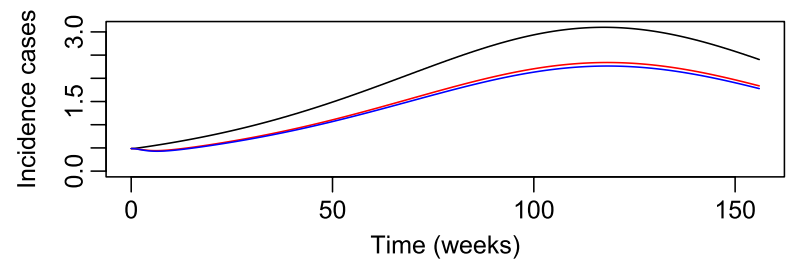

(a)

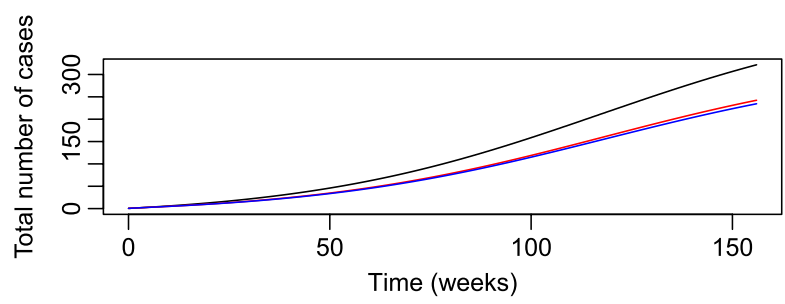

(b)

Fig. 6. Numerical simulations for (a) incidence cases (in weeks) and (b) total number of dengue cases within the flats for $P=0.6610$ where the black line, red line and blue line represent $P^{*}=$ $0, P^{*}=0.6871$ and $P^{*}=0.7789$, respectively, over a period of three years.
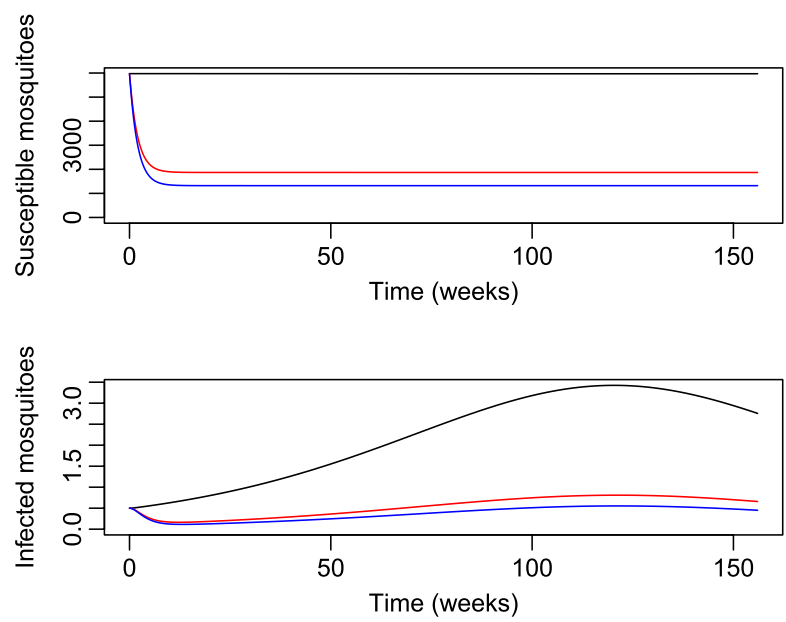

Fig. 7. Numerical simulation for the dynamical behavior of Aedes mosquitoes within the flats for $P=0.6610$ where the black line, red line and blue line represent $P^{*}=0, P^{*}=0.6871$ and $P^{*}=0.7789$ respectively, over a period of three years.

MHSs in the blocks of flats, the number of susceptible and infected have reduced. Again similarly, the number of incidence cases as well as the total number of dengue cases are reduced as a result of the MHSs as shown in Fig. 6. The $P^{*}$ value that performs the best by having the lowest number of infected mosquitoes is when we have 552 MHSs inside the flats, namely when $P^{*}=0.7789$. 


\subsection{Effect of $P^{*}$ on spread of dengue in flats}

In order for us to examine the effect that each $P^{*}$ has on the spread of dengue within the flats, we will now look at the mean values for each variable. The results for various $P$ values and $P^{*}$ values are given in Tables [6, [8] 10] and 12] where their corresponding endemic equilibrium values are given in Tables [7, 9, [1] and 13. respectively.

From Table 6 where $P=0.1280$, we can see that as $P^{*}$ increases, the number of infected individuals, incidence cases, susceptible mosquitoes, infected mosquitoes and latent mosquitoes all decrease accordingly. If we compare this to the results given in Table 8 where $P=0.3190$, then we can see that although those variables still decrease as $P^{*}$ increases, the relative change is not as significant compared to when $P=0.1280$.

Table 6. Mean values for variables within the flats for $P=0.1280$, where $P^{*}=0$ : No MHSs, $P^{*}=0.6871: 344$ MHSs and 356 ovitraps and $P^{*}=0.7789: 552$ MHSs and 356 ovitraps to 3 d.p over one year.

\begin{tabular}{cccccc}
\hline \multicolumn{5}{c}{$P=0.1280$} \\
\hline$P^{*}$ values & $S_{H_{2}}(t)$ & $I_{H_{2}}(t)$ & $R_{H_{2}}(t)$ & Incidence cases & Total number of cases \\
\hline 0 & $2,946.550$ & 0.912 & 252.538 & 0.933 & 48.984 \\
0.6871 & $2,960.740$ & 0.209 & 239.051 & 0.206 & 11.170 \\
0.7789 & $2,961.215$ & 0.190 & 238.595 & 0.186 & 10.151 \\
\hline
\end{tabular}

\begin{tabular}{cccc}
\hline$P^{*}$ values & $S_{v_{2}}(t)$ & $L_{v_{2}}(t)$ & $I_{v_{2}}(t)$ \\
\hline 0 & $5,973.959$ & 0.745 & 0.966 \\
0.6871 & $2,027.497$ & 0.077 & 0.101 \\
0.7789 & $1,500.067$ & 0.059 & 0.078 \\
\hline
\end{tabular}

Table 7. Endemic equilibrium values for variables within the flats for $P=0.1280$, where $P^{*}=0$ : No MHSs, $P^{*}=0.6871: 344 \mathrm{MHSs}$ and 356 ovitraps and $P^{*}=0.7789: 552 \mathrm{MHSs}$ and 356 ovitraps to 3 d.p.

\begin{tabular}{ccrrcc}
\hline \multicolumn{6}{c}{$P=0.1280$} \\
\hline$P^{*}$ values & $S_{H_{2}}(t)$ & $I_{H_{2}}(t)$ & $R_{H_{2}}(t)$ & $\begin{array}{c}\text { Incidence } \\
\text { cases }\end{array}$ & $\begin{array}{c}\text { Total number of } \\
\text { cases per year }\end{array}$ \\
\hline 0 & $2,753.155$ & 0.115 & 446.730 & 0.115 & 5.958 \\
0.6871 & $3,106.745$ & 0.024 & 93.231 & 0.024 & 1.243 \\
0.7789 & $3,117.158$ & 0.021 & 82.821 & 0.021 & 1.105 \\
\hline
\end{tabular}

\begin{tabular}{cccc}
\hline$P^{*}$ values & $S_{v_{2}}(t)$ & $L_{v_{2}}(t)$ & $I_{v_{2}}(t)$ \\
\hline 0 & $5,975.466$ & 0.098 & 0.127 \\
0.6871 & $1,869.773$ & 0.006 & 0.008 \\
0.7789 & $1,321.212$ & 0.004 & 0.005 \\
\hline
\end{tabular}


Table 8. Mean values for variables within the flats for $P=0.3190$, where $P^{*}=0$ : No MHSs, $P^{*}=0.6871: 344$ MHSs and 356 ovitraps and $P^{*}=0.7789: 552$ MHSs and 356 ovitraps to 3 d.p over one year.

\begin{tabular}{|c|c|c|c|c|c|c|}
\hline \multicolumn{7}{|c|}{$P=0.3190$} \\
\hline$P^{*}$ values & $S_{H_{2}}(t)$ & $I_{H_{2}}(t)$ & $R_{H_{2}}(t)$ & \multicolumn{2}{|c|}{ Incidence cases } & Total number of cases \\
\hline 0 & $2,946.55$ & 0.912 & 252.538 & \multicolumn{2}{|c|}{0.933} & 48.984 \\
\hline 0.6871 & $2,956.848$ & 0.404 & 242.748 & \multicolumn{2}{|c|}{0.407} & 21.667 \\
\hline 0.7789 & $2,957.457$ & 0.376 & 242.167 & \multicolumn{2}{|c|}{0.379} & 20.178 \\
\hline & & $P^{*}$ values & $S_{v_{2}}(t)$ & $L_{v_{2}}(t)$ & $I_{v_{2}}(t)$ & \\
\hline & & 0 & $5,973.959$ & 0.745 & 0.966 & \\
\hline & & 0.6871 & $2,027.385$ & 0.126 & 0.164 & \\
\hline & & 0.7789 & $1,499.99$ & 0.093 & 0.121 & \\
\hline
\end{tabular}

Table 9. Endemic equilibrium values for variables within the flats for $P=0.3190$, where $P^{*}=0$ : No MHSs, $P^{*}=0.6871: 344$ MHSs and 356 ovitraps and $P^{*}=0.7789: 552$ MHSs and 356 ovitraps to 3 d.p.

\begin{tabular}{cllccc}
\hline \multicolumn{5}{c}{$P=0.3190$} \\
\hline$P^{*}$ values & \multicolumn{1}{c}{$S_{H_{2}}(t)$} & $I_{H_{2}}(t)$ & $R_{H_{2}}(t)$ & $\begin{array}{c}\text { Incidence } \\
\text { cases }\end{array}$ & $\begin{array}{c}\text { Total number of } \\
\text { cases per year }\end{array}$ \\
\hline 0 & $2,753.155$ & 0.115 & 446.730 & 0.115 & 5.958 \\
0.6871 & $2,997.889$ & 0.052 & 202.059 & 0.052 & 2.695 \\
0.7789 & $3,013.21$ & 0.048 & 186.742 & 0.048 & 2.491 \\
\hline
\end{tabular}

\begin{tabular}{cccc}
\hline$P^{*}$ values & \multicolumn{1}{c}{$S_{v_{2}}(t)$} & $L_{v_{2}}(t)$ & $I_{v_{2}}(t)$ \\
\hline 0 & $5,975.446$ & 0.098 & 0.127 \\
0.6871 & $1,869.755$ & 0.014 & 0.018 \\
0.7789 & $1,321.20$ & 0.009 & 0.012 \\
\hline
\end{tabular}

Table 10. Mean values for parameters within the flats for $P=0.4770$, where $P^{*}=0$ : No MHSs, $P^{*}=0.6871: 344$ MHSs and 356 ovitraps and $P^{*}=0.7789: 552$ MHSs and 356 ovitraps to 3 d.p over one year.

\begin{tabular}{|c|c|c|c|c|c|c|}
\hline \multicolumn{7}{|c|}{$P=0.4770$} \\
\hline$P^{*}$ values & $S_{H_{2}}(t)$ & $I_{H_{2}}(t)$ & $R_{H_{2}}(t)$ & \multicolumn{2}{|c|}{ Incidence cases } & Total number of cases \\
\hline 0 & $2,946.55$ & 0.912 & 252.538 & \multicolumn{2}{|c|}{0.933} & 48.984 \\
\hline 0.6871 & $2,954.022$ & 0.545 & 245.434 & \multicolumn{2}{|c|}{0.553} & 29.228 \\
\hline \multirow[t]{5}{*}{0.7789} & $2,954.608$ & 0.517 & 244.875 & \multicolumn{2}{|c|}{0.524} & 27.740 \\
\hline & & $P^{*}$ values & $S_{v_{2}}(t)$ & $L_{v_{2}}(t)$ & $I_{v_{2}}(t)$ & \\
\hline & & 0 & $5,973.959$ & 0.745 & 0.966 & \\
\hline & & 0.6871 & $2,027.304$ & 0.162 & 0.210 & \\
\hline & & 0.7789 & $1,499.932$ & 0.118 & 0.153 & \\
\hline
\end{tabular}


Table 11. Endemic equilibrium values for variables within the flats for $P=0.4770$, where $P^{*}=0$ : No MHSs, $P^{*}=0.6871: 344$ MHSs and 356 ovitraps and $P^{*}=0.7789: 552$ MHSs and 356 ovitraps to 3 d.p.

\begin{tabular}{cccccc}
\hline \multicolumn{6}{c}{$P=0.4770$} \\
\hline$P^{*}$ values & $S_{H_{2}}(t)$ & $I_{H_{2}}(t)$ & $R_{H_{2}}(t)$ & $\begin{array}{c}\text { Incidence } \\
\text { cases }\end{array}$ & $\begin{array}{c}\text { Total number of } \\
\text { cases per year }\end{array}$ \\
\hline 0 & $2,753.155$ & 0.115 & 446.730 & 0.115 & 5.958 \\
0.6871 & $2,925.803$ & 0.070 & 274.127 & 0.070 & 3.656 \\
0.7789 & $2,940.294$ & 0.067 & 259.640 & 0.067 & 3.463 \\
\hline
\end{tabular}

\begin{tabular}{cccc}
\hline$P^{*}$ values & $S_{v_{2}}(t)$ & $L_{v_{2}}(t)$ & $I_{v_{2}}(t)$ \\
\hline 0 & $5,975.446$ & 0.098 & 0.127 \\
0.6871 & $1,869.744$ & 0.019 & 0.024 \\
0.7789 & $1,321.192$ & 0.013 & 0.016 \\
\hline
\end{tabular}

Table 12. Mean values for variables within the flats for $P=0.6610$, where $P^{*}=0$ : No MHSs, $P^{*}=0.6871: 344$ MHSs and 356 ovitraps and $P^{*}=0.7789: 552$ MHSs and 356 ovitraps to 3 d.p over one year.

\begin{tabular}{cccccc}
\hline \multicolumn{5}{c}{$P=0.6610$} \\
\hline$P^{*}$ values & $S_{H_{2}}(t)$ & $I_{H_{2}}(t)$ & $R_{H_{2}}(t)$ & Incidence cases & Total number of cases \\
\hline 0 & $2,946.55$ & 0.912 & 252.538 & 0.933 & 48.984 \\
0.6871 & $2,951.10$ & 0.689 & 248.211 & 0.702 & 36.991 \\
0.7789 & $2,951.552$ & 0.667 & 247.781 & 0.680 & 35.822 \\
\hline
\end{tabular}

\begin{tabular}{cccc}
\hline$P^{*}$ values & $S_{v_{2}}(t)$ & $L_{v_{2}}(t)$ & $I_{v_{2}}(t)$ \\
\hline 0 & $5,973.959$ & 0.745 & 0.966 \\
0.6871 & $2,027.22$ & 0.198 & 0.257 \\
0.7789 & $1,499.871$ & 0.145 & 0.188 \\
\hline
\end{tabular}

Table 13. Endemic equilibrium values for variables within the flats for $P=0.6610$, where $P^{*}=0$ : No MHSs, $P^{*}=0.6871: 344$ MHSs and 356 ovitraps and $P^{*}=0.7789: 552$ MHSs and 356 ovitraps to 3 d.p.

\begin{tabular}{cccccc}
\hline \multicolumn{5}{c}{$P=0.6610$} \\
\hline$P^{*}$ values & $S_{H_{2}}(t)$ & $I_{H_{2}}(t)$ & $R_{H_{2}}(t)$ & $\begin{array}{c}\text { Incidence } \\
\text { cases }\end{array}$ & $\begin{array}{c}\text { Total number of } \\
\text { cases per year }\end{array}$ \\
\hline 0 & $2,753.155$ & 0.115 & 446.730 & 0.115 & 5.958 \\
0.6871 & $2,855.752$ & 0.088 & 344.160 & 0.088 & 4.590 \\
0.7789 & $2,866.451$ & 0.086 & 333.463 & 0.086 & 4.447 \\
\hline
\end{tabular}

\begin{tabular}{cccc}
\hline$P^{*}$ values & $S_{v_{2}}(t)$ & $L_{v_{2}}(t)$ & $I_{v_{2}}(t)$ \\
\hline 0 & $5,975.446$ & 0.098 & 0.127 \\
0.6871 & $1,869.733$ & 0.024 & 0.031 \\
0.7789 & $1,321.184$ & 0.016 & 0.021 \\
\hline
\end{tabular}


From Tables 6 8, 10 and 12, for a higher value of $P$, in other words, individuals are getting bitten more when going outside at certain time intervals, they are more likely to bring in new infections into the flats. Although using the MHSs within the blocks of flats reduced the number of infected individuals as well as the number of incidence cases, the effect decreases as $P$ increases. This is to be expected as smaller $P$ values represent the individuals spending relatively more time in the flats so the effect of the MHSs is bigger (for the same value of $P^{*}$ ).

Nonetheless, it is important to note that the MHSs are effective in reducing and controlling the spread of dengue within the blocks of flats both in the short run and in the long run.

\subsection{Starting at endemic equilibrium}

In this section, we will continue to examine the effect of having different number of MHSs within the flats when our population starts at the endemic equilibrium (EE) in the absence of MHSs. Let us define the EE initial values as follows:

$$
\begin{aligned}
& S_{H_{1}}(0)=1,479,821, \quad I_{H_{1}}(0)=61.569, \quad R_{H_{1}}(0)=240,117.60 \text {, } \\
& S_{v_{1}}(0)=3,211,802, \quad L_{v_{1}}(0)=52.546, \quad I_{v_{1}}(0)=68.171, \\
& S_{H_{2}}(0)=2,753.155, \quad I_{H_{2}}(0)=0.115, \quad R_{H_{2}}(0)=446.730, \\
& S_{v_{2}}(0)=5,975.446, \quad L_{v_{2}}(0)=0.098, \quad I_{v_{2}}(0)=0.127,
\end{aligned}
$$

where all the parameter values are defined as before. Note that the above EE initial values are obtained from numerical simulations where $P^{*}=0$.

The effects of introducing the MHSs into the flats when the population system is at endemic equilibrium are illustrated in both Tables 14 and 15] for when $P=0.1280$ and $P=0.6610$, respectively. For both cases, we noticed a drastic decrease in the number of infected individuals within the blocks of flats when we first introduced the MHSs (82.36\% decrease for $P=0.1280$ and $26.02 \%$ decrease for $P=0.6610)$. When we increase the number of MHSs from 344 to 552 , although the number of infected individuals have been reduced further, the relative changes are not as significant (a further decrease of $9.93 \%$ for $P=0.1280$ and $3.36 \%$ for $P=0.6610$ ).

Table 14. Variable values within the flats for $P=0.1280$, where $P^{*}=0.6871: 344$ MHSs and 356 ovitraps and $P^{*}=0.7789: 552 \mathrm{MHSs}$ and 356 ovitraps to 3 d.p over a period of 52 weeks with initial values starting at EE.

\begin{tabular}{cccccccc}
\hline \multicolumn{7}{c}{$P=0.1280$} \\
\hline $\begin{array}{c}P^{*} \\
\text { values }\end{array}$ & $S_{H_{2}}(t)$ & $I_{H_{2}}(t)$ & $R_{H_{2}}(t)$ & $\begin{array}{c}\text { Incidence } \\
\text { cases }\end{array}$ & $S_{v_{2}}(t)$ & $L_{v_{2}}(t)$ & $I_{v_{2}}(t)$ \\
\hline 0.6871 & $2,757.571$ & 0.020 & 442.409 & 0.020 & $1,869.775$ & 0.005 & 0.007 \\
0.7789 & $2,757.706$ & 0.018 & 442.276 & 0.018 & $1,321.213$ & 0.003 & 0.004 \\
\hline
\end{tabular}


Table 15. Variable values within the flats for $P=0.6610$, where $P^{*}=0.6871: 344$ MHSs and 356 ovitraps and $P^{*}=0.7789$ : 552 MHSs and 356 ovitraps to 3 d.p over a period of 52 weeks with initial values starting at $\mathrm{EE}$.

\begin{tabular}{cccccccc}
\hline \multicolumn{7}{c}{$P=0.6610$} \\
\hline$P^{*}$ & $S_{H_{2}}(t)$ & $I_{H_{2}}(t)$ & $R_{H_{2}}(t)$ & $\begin{array}{c}\text { Incidence } \\
\text { cases }\end{array}$ & $S_{v_{2}}(t)$ & $L_{v_{2}}(t)$ & $I_{v_{2}}(t)$ \\
values & & & & & & & \\
\hline 0.6871 & $2,754.569$ & 0.085 & 445.346 & 0.085 & $1,869.735$ & 0.023 & 0.029 \\
0.7789 & $2,754.708$ & 0.082 & 445.210 & 0.082 & $1,321.185$ & 0.015 & 0.020 \\
\hline
\end{tabular}

Nonetheless, it is reassuring to see that the MHSs are effective even when the population system is already at endemic equilibrium level.

\subsection{Endemic equilibrium}

Another way in which we can examine the effect of using the MHSs within the flats and later deploy them throughout Malaysia would be to look at the endemic equilibrium (EE) level. In this section, we will compare the endemic equilibrium level when we have no MHSs within the flats and when we have the MHSs within the three blocks of flats. In this section, we will set $P=0.6610$ where this $P$ value represents people spending time outdoors between 6 am and $7.45 \mathrm{pm}$.

For the case when we have no MHSs in the flats, in other words when $P^{*}=0$, the endemic equilibrium value for the number of infected individuals in KL and in the flats are 61.569 and 0.115 , respectively to 3 d.p.

For the case when we have introduced 552 MHSs inside the three blocks of flats, we have that the endemic equilibrium points for the number of infected individuals in $\mathrm{KL}$ and in the flats are 61.569 and 0.086 , respectively to 3 d.p.

From the above results, we can see that although by having the MHSs inside the flats has reduced the endemic equilibrium level for the number of infected individuals within the flats by around $25 \%$, we notice an insignificant change in the endemic equilibrium level for the number of infected individuals in KL. This indicates that although the MHSs have successfully reduced the number of dengue cases within the flats, in order to achieve extinction within the whole of KL and thus later to the whole of Malaysia, more MHSs would need to be deployed in more places throughout KL.

Table 16 illustrates the effect of having different $P$ and $P^{*}$ values on the number of incidence cases at EE.

From Table 17 we can see clearly that as $P$ increases, the percentage reduction in the average number of incidence cases from when we have 344 MHSs within the flats to 552 MHSs decreases. In other words, if individuals who reside within the blocks of flats go outdoors the majority of the time and thus get bites from outdoors, the effect from increasing the number of the MHSs within the flats gets reduced. Putting this in a non-mathematical context, if the majority of the people resident in the blocks of flats are in KL between 6 am to $7.45 \mathrm{pm}$, then the difference between 
Table 16. Number of of incidence cases per week at EE for different number of MHSs within the flats for different proportions of time spent outdoors.

\begin{tabular}{cccc}
\hline$P$ values & 0 MHSs & $344 \mathrm{MHSs}$ & $554 \mathrm{MHSs}$ \\
\hline 0.1280 & 0.115 & 0.024 & 0.021 \\
0.3190 & 0.115 & 0.052 & 0.048 \\
0.4770 & 0.115 & 0.070 & 0.067 \\
0.6610 & 0.115 & 0.088 & 0.086 \\
\hline
\end{tabular}

Table 17. Percentage reduction in the average number of incidence cases for various values of $P$ within the flats at EE.

\begin{tabular}{ccc}
\hline$P$ values & $P^{*}=0-0.6871$ & $P^{*}=0.6871-0.7789$ \\
\hline 0.1280 & $79.13 \%$ & $12.50 \%$ \\
0.3190 & $54.78 \%$ & $7.69 \%$ \\
0.4770 & $39.13 \%$ & $4.29 \%$ \\
0.6610 & $23.48 \%$ & $2.27 \%$ \\
\hline
\end{tabular}

using $344 \mathrm{MHSs}$ and $552 \mathrm{MHSs}$ is only $2.27 \%$ difference in the average number of incidence cases with an impact ratio of only 0.0227 .

\section{Discussion and Conclusion}

In this paper, we have used the classic Ross-Macdonald model ${ }^{6}$ to describe the spread of dengue in Malaysia between human and Aedes mosquitoes. Note that the classic Ross-Macdonald model ${ }^{6}$ was based on analyzing the Anopheles mosquito, which is responsible for transmitting malaria. However, over the past years, many researchers have also used the classic Ross-Macdonald model $\sqrt{6}$ to describe the spread of other vector-borne diseases, most specifically on dengue, for example. $823+25$

By using the MHSs trial data collected in the three blocks of flats in Selangor, ${ }^{7}$ we have performed different analyses to investigate the effect of using different numbers of MHSs on the number of dengue cases in the trial site. In Sec. [3, we have mathematically estimated the number of potential Aedes breeding sites which will help us in deciding the correct number of MHSs that we would need to deploy to control the spread of dengue. From the numerical simulations produced throughout this paper, it is clear that using the MHS does indeed reduce the number of susceptible and infected mosquitoes. As a result, the MHSs have effectively reduced the number of incidence cases within the trial sites and thus reduced the number of dengue cases within that area. Our results have suggested that by using the MHSs in a high-rise condominium environment, the number of dengue cases have reduced, which is very promising.

It is important to note, however, that despite the effectiveness in reducing the number of dengue cases within the blocks of flats, it shows very little effect in reducing the number of dengue cases in KL as a whole. This is to be expected as 
the effect from the three blocks of flats is not significant enough to cause an overall impact to KL especially when dengue is endemic in Malaysia. This implies that, in order to achieve the effect that we experienced in the three blocks of flats, we would need to deploy MHSs in other areas in KL.

\section{Acknowledgments}

This work was supported by the Dengue Tech Challenge 2016 grant (Application Reference DTC 16022), under the Newton-Ungku Omar Fund partnership. The grant is funded by the UK Department for Business, Energy and Industrial Strategy and Malaysian Industry-Government Group for High Technology (MIGHT) and delivered by the British Council and PlaTCOM Ventures. The authors are grateful to One Team Networks Sdn Bhd, in Malaysia, as our principal collaborator in Malaysia for the Dengue Tech Challenge 2016 and for providing the data.

DG is grateful to the Science Without Borders Program, Brazil, for a Special Visiting Fellowship (CNPq grant 30098/2014-7), with Professor E. Massad, School of Applied Mathematics, Fundacao Getulio Vargas, Rio de Janeiro, Brazil, and to the Leverhulme Trust for support from a Leverhulme Research Fellowship (RF2015-88).

\section{References}

1. Mutebi J, Gianella A, Travassos da Rosa A, Tesh RB, Barrett ADT, Higgs S, Yellow fever virus infectivity for Bolivian Aedes aegypti mosquitoes, Emerg Inf Dis 10(9):1657-1660, 2004.

2. World Health Organization. Dengue control, 2018, Available at http://www.who.int/ denguecontrol/disease/en/.

3. Centers for Disease Control and Prevention. Zika virus, 2018, Available at https:// www.cdc.gov/zika/vector/range.html.

4. Packierisamy PR, Ng C, Dahlui M, Inbaraj J, Balan VK, Halasa YA, Shepard DS, Cost of dengue vector control activities in Malaysia, Amer J Trop Med Hyg 93(5):1020-1027, 2015.

5. Lee HL, Rohani A, Khadri MS, Nazni WA, Rozilawati H, Nurulhusna AH, Nor Afizah AH, Roziah A, Rosilawati R, Tech CH, Dengue vector control in Malaysia Challenges and recent advances, Int Med J Malaysia 14(1):11-15, 2015.

6. Macdonald G, The analysis of equilibrium in malaria, Trop Dis Bull 49(9):813-829, 1952.

7. Nazni WA, Teoh GN, Oreenaiza N, Farah H, Suhana O, Chandru A, Khairul Asuad M, Norazizah A, Azman M, Muhd. Tarmimie MJ, Mohd. Afig MS, Wan Nur Alia Fatin WZ, Topek O, Liang Y, Greenhalgh D, Lees R, Lee HL, Suppression of containerbreeding Aedes and interruption of dengue transmission in high-rise condominia using Pyriproxifen treated dissemination traps, Unpublished manuscript.

8. Massad E, Coutinho FAB, Burattini MN, Amaku M, Estimation of $R_{0}$ from the initial phase of an outbreak of a vector-borne infection, Trop Med Int Health 15(1):120-126, 2010 .

9. Massad E, Coutinho FAB, Burattini MN, Lopez LR, The risk of yellow fever in a dengue-infested area, Trans Roy Soc Trop Med Hyg 95:370-374, 2001. 
10. The World Bank. Data indicators, 2017, Available at https://data.worldbank. org/indicator.

11. Singapore National Environment Agency. Dengue \& zika-Prevent Aedes mosquito breeding — Aedes mosquito, 2018, Available at https://www.nea.gov.sg/denguezika/prevent-aedes-mosquito-breeding/aedes-mosquito.

12. Andraud M, Hens N, Marais C, Beutels P, Dynamic epidemiological models for dengue transmission: A systematic review of structural approaches, PLoS One 7(11):e49085, 2012 .

13. Liew SM, Khoo EM, Ho BK, Lee YK, Omar M, Ayadurai V, Yusoff FM, Suli Z, Mudin RN, Goh PP, Chinna K, Dengue in Malaysia: Factors associated with dengue mortality from a national registry, PLoS One 11(6):e0157631, 2016, Available at doi:10.1371/journal.pone.0157631.

14. Derouich M, Boutayeb A, Dengue fever: Mathematical modelling and computer simulation, Appl Math Comput 177(2):528-544, 2006.

15. Scanpap (Asia Pacific) Pte Ltd and One Team Networks Sdn Bhd, Project report for field trail conducted from 04-Dec-2015 till 27-Jan-2016, 2016, Unpublished report.

16. Lee C, Vythilingam I, Chong C, Razak M, Tan C, Liew C, Pok K, Ng L, Gravitraps for management of dengue clusters in Singapore, Amer J Trop Med Hyg 88(5):888-892, 2013.

17. Department of Statistics Malaysia. Official portal — Federal territory of Kuala Lumpur, 2017, Available at https://www.dosm.gov.my.

18. Myhealth Dengue Official Portal of Malaysian Health Ministry. History and epidemiology of dengue, 2017, Available at http://denggi.myhealth.gov.my/history-andepidemiology-of-dengue/?lang=en.

19. World Health Organization. Dengue control — Dengue/severe dengue frequently asked questions - Who spreads dengue and severe dengue?, 2017, Available at http://www.who.int/denguecontrol/faq/en/index5.html.

20. Official Portal of Ministry of Health Malaysia, 2018, Available at http://www.moh. gov.my/index.php/database_stores/store_view/17.

21. Olivera-Botello G, Coudeville L, Fanouillere K, Guy B, Chambonneau L, Noriega F, Jackson N, The CYD-TDV Vaccine Group. Tetravalent dengue vaccine reduces symptomatic and asymptomatic dengue virus infections in healthy children and adolescents aged 2-16 Years in Asia and Latin America, J Inf Dis 214(7):994-1000, 2016.

22. Wilder-Smith A, Chen LH, Wilson ME, Threat of dengue to blood safety in dengueendemic countries, Emerg Inf Dis 15(1):8-11, 2009.

23. Amaku M, Azevedo F, Burattini MN, Coelho GE, Coutinho FA, Greenhalgh D, Lopez LF, Motitsuki RS, Wilder-Smith A, Massad E, Magnitude and frequency variations of vector-borne infection outbreaks using the Ross-Macdonald model: Explaining and predicting outbreaks of dengue fever, Epidemiol Inf 144(16):3435-3460, 2016.

24. Cheng Q, Jing Q, Spear RC, Marshall JM, Yang Z, Gong P, Climate and the timing of imported cases as determinants of the dengue outbreak in Guangzhou, 2014: Evidence from a mathematical model, PLoS Negl Trop Dis 10(2):e0004417, 2016.

25. Schmidt WP, Suzuki M, Thiem VD, White RG, Tsuzuki A, Yoshida LM, Yanai H, Haque U, Anh DD, Ariyoshi K, Population density, water supply, and the risk of dengue fever in Vietnam: Cohort study and spatial analysis, PLoS Med 8(8):e1001082, 2011 . 